\title{
Research on Automatic Discovery and Topology Rendering of Network Devices
}

\author{
Wu Wenxuan ${ }^{1}$, Wu Fei ${ }^{1}$, Cai Yuxiang ${ }^{2}$, Zhang Huaigang ${ }^{3}$, Lin Wei ${ }^{3, a}$ \\ ${ }^{1}$ State Grid Fujian Electric Power Company, Fuzhou 350003, China \\ ${ }^{2}$ State Grid Fujian information\&telecommunication company, Fuzhou,350003, China \\ ${ }^{3}$ Fujian Yirong Information Technology Co.Ltd, Fuzhou,350003,China \\ aninwei8868@163.com
}

Keywords: network device; automatic discovery technology; topology rendering technology

\begin{abstract}
The revolution in social production technology in Internet era has brought increasingly important influence. This paper analyzes the automatic discovery and topology rendering of network device, which are important target technology in the current and future network device development and application and attract extensive attention from various fields because of the superiority. Therefore, research on the relevant content has some positive significance.
\end{abstract}

\section{Introduction}

Automatic discovery technology and topology rendering technology are important for network device tracking. The so-called automatic discovery technology can meet the demands of network resource allocation and connection in small-scale environment, and save the trouble of manual operation. Topology rendering technology can realize the rough division and rendering of target object, so that the related content can be displayed intuitively. The topology in automatic discovery technology can undoubtedly increase the work efficiency, which is an important pursuit of the information age. Therefore, it is necessary to analyze the relevant content.

\section{Automatic discovery technology of network devices}

\section{Concepts and advantages of automatic discovery technology}

The current network devices, whether wireless or wired, need to find the target network and the IP protocol to realize network connection, which is generally processed manually. Take the wireless network commonly used as an example. After the smart phone discovers the network, the password needs to be entered manually to achieve the connection. The so-called automatic discovery technology does not mean simple discovery of connection objects, but refers to the automatic connection after discovery, which enhances the efficiency of related work in an automatic configuration way. The advantages of this technology are reflected in its superior maneuverability and efficiency. Without personnel operation, it means that the maximum error rate is reduced, and the automatic execution ensures the efficiency. The advantages and disadvantages compared with traditional technology are as shown in Table 1. 
Table 1 Comparison between automatic discovery technology and traditional technology

\begin{tabular}{ccccc}
\hline $\begin{array}{c}\text { Technology } \\
\text { type }\end{array}$ & $\begin{array}{c}\text { Connection } \\
\text { type }\end{array}$ & $\begin{array}{c}\text { Connection } \\
\text { speed }\end{array}$ & $\begin{array}{c}\text { Resource } \\
\text { allocation }\end{array}$ & $\begin{array}{c}\text { Application } \\
\text { prospect }\end{array}$ \\
\hline $\begin{array}{c}\text { Automatic } \\
\text { discovery }\end{array}$ & Automatic & Maximize & $\begin{array}{c}\text { Intelligent } \\
\text { allocation }\end{array}$ & Extensive \\
$\begin{array}{c}\text { technology } \\
\text { Traditional } \\
\text { technology }\end{array}$ & Manual & Unknown & $\begin{array}{c}\text { Unknown } \\
\text { allocation }\end{array}$ & General \\
\hline
\end{tabular}

At present, various research institutions are trying to study automatic discovery technology, emergence and popularization of which will bring another great reform in the information age.

\section{Key technology of automatic discovery technology}

Combined with the existing technology, we can basically understand the working principle and process of automatic discovery technology. In the current network connection, it is usually necessary to discover the target, analyze the domain name, and then achieve the connection through the IP protocol. The core of this process is the legitimacy of domain names. Under the existing technical conditions, the source address side generally audits the IP protocol, and analyzes the domain name. The target device cannot realize the connection through the formal channel and the original address in the illegal situation. This is the core problem to be solved in automatic discovery technology.

Zero configuration technology has gained importance as a means of realizing automatic discovery technology. The so-called zero configuration refers to the zero namual configuration, and the network device realizes communication by its function. In the current theory, the main components of the zero allocation technology include three aspects: IP automatic allocation, domain name automatic acquisition and service discovery. The specific functions of the three aspects are as shown in Table 2.

Table 2 Main function of the three components of zero configuration technology

\begin{tabular}{|l|l|c|c|c|}
\hline \multicolumn{1}{|c|}{$\begin{array}{c}\text { Name of } \\
\text { component }\end{array}$} & Function & Value & Practicability & $\begin{array}{c}\text { Implementation } \\
\text { difficulty }\end{array}$ \\
\hline $\begin{array}{l}\text { IP Automatic } \\
\text { Allocation }\end{array}$ & $\begin{array}{l}\text { IP Access to legally } \\
\text { available IP }\end{array}$ & High & Middle & Middle \\
\hline $\begin{array}{l}\text { Automatic } \\
\text { acquisition of } \\
\text { domain name }\end{array}$ & $\begin{array}{l}\text { Automatically filter the } \\
\text { available domains }\end{array}$ & Middle & Middle & High \\
\hline Service discovery & $\begin{array}{l}\text { Discover and connect } \\
\text { services }\end{array}$ & Middle & High & Middle \\
\hline
\end{tabular}

Automatic allocation of IP needs to be achieved by means of the target object. The basic mode is as follows: the target object of zero configuration sends a service request to all hosts that can provide service. If a service request is not provided by the host, a random IP address will be generated, and other hosts will be requested for availability. If available, it is a native IP address. If not available, it will be sent again until service is received[1].

The main point of the link is whether the request for the zero configuration target object is received. If the response is sent in the first step, $t$ the IP can be obtained directly without subsequent action.

It is assumed that the service is responded, and the second issue involved is the acquisition of domain name. In the zero configuration mode, the domain name is automatically acquired, and the core of the link is to determine the uniqueness of the domain name. The IP address is changeable, mobile IP is countless, and a lot of IP addresses are even invalid. Therefore, when trying to obtain a domain name, three data will be randomly generated and sent to the service provider until the domain name is passed. In the zero configuration mode, domain names and IP are bidirectional only. That is, the IP address can be found through the domain name and the domain name can also be determined through the IP address. This is a self-protection of automatic discovery technology in intelligent mode. The basic mode of this link is shown in Table 3. 
Table 3 Automatic acquisition mode of domain name

\begin{tabular}{lll}
\hline Work content & Whether passed by the host? & Follow-up work \\
\hline Send random data & No & Continue sending \\
Send random data & Yes & Connect \\
\hline
\end{tabular}

The core of the service discovery link is the host that provides the service. The technical requirements of this link are the lowest in the three links, and the existing technical conditions can support the realization of this link. It can be considered as the derivative product of IP automatic allocation and domain name automatic acquisition. However, the practicability of this link is the highest. Without the service discovery link, the IP automatic allocation and the domain name automatic acquisition become meaningless. The basic work pattern is as follows: When the IP is automatically assigned and the domain name is automatically obtained, the host provides service to the connection object, which selects the service type independently. For example, in closed conference, the host provides the service like data downloads, online reading, and font enhancement, and other services are selected by the object target. If the effective connection can be realized bilaterally, there will be no major problems in the service discovery process.

\section{Topology rendering technology of network devices}

\section{Concept and advantage of topology rendering technology}

Topology rendering, as the name suggests, refers to a rendering by topological concept. The objects are rendered according to the category and performance. Such images generally do not take into consideration the specific size of each sub object.

Topology rendering is a relatively intelligent rendering method, and its advantage is that it can render the sub-network infinitely. For instance, the management of a company includes personnel, finance, logistics. After the topology renders the three projects, the debts, profits, loans, and other projects under the financial sub network are rendered, so that the whole system is visually displayed in the form of images, until the rendering of all subnet is finished. The advantages and disadvantages of topological rendering and traditional rendering are shown in Table 4.

Table 4 Comparison of topological rendering and traditional rendering

\begin{tabular}{clll}
\hline Rendering method & Complete rendering & Intelligent rendering & Operability \\
\hline Topological rendering & Yes & Yes & High \\
Traditional rendering & No & No & Low \\
\hline
\end{tabular}

\section{Topology rendering technology in automatic discovery environment}

In automatic discovery environment, topology rendering technology has four basic elements, that is, database model, automatic rendering module, adjustment module and device link module. The corresponding functions are as shown in Table 5. 
Table 5 Four elements and corresponding functions of topological rendering

\begin{tabular}{lll}
\hline Element & Function & Importance \\
\hline Database model & Provide rendering material & High \\
Automatic rendering module & Implement rendering work & High \\
Adjustment module & Adjust various details & Middle \\
Device link module & Check rendering contents & Middle \\
\hline
\end{tabular}

Database model and automatic rendering module are the key to topology rendering. The model provided by the database is the basis for topological classification and rendering. In general, the database model should cover the head and a number of subhead, namely, a sub network. Under the existing conditions, after the database is built, ADO can implement the connection, which is convenient for data retrieval.

In the automatic discovery mode, in the device with database and automatic rendering module, the rendering module can automatically analyze and render the data by applying the topology principle. The basic step is to first implement the connection through ADO, and the rendering module can read the contents of the data. The part to be rendered needs to be temporarily stored through the link and then the link is closed. The part is transferred out of the link and inserted into the rendering module for analysis and rendering. The building of the rendering module is based on the work required. For instance, the default paper size is $20 \mathrm{~cm} * 20 \mathrm{~cm}$, when the module is set, we should pay attention to the proportion to avoid the problems like the image is too small, not clear, too large, or incomplete. This link is as shown in Table 6.

Table 6 Work of automatic rendering module

\begin{tabular}{lll}
\hline Function module & Function & Whether support intelligence \\
\hline Search module & Search database & Yes \\
Check module & Check rendering objects & Yes \\
Retrieval module & Retrieve rendering content & Yes \\
Rendering module & Analyze and render & Yes \\
\hline
\end{tabular}

After the rendering is completed, necessary adjustment should be implemented by adjusting the module. The main object of the adjustment is whether the connection between the modules is correct. In topology principle, the connection between the modules is the key point. For example, the financial content of an enterprise includes loan, financing, utilization, and recycling, etc. In topology theory, we must first make clear the relationship between these five targets. For example, the use link is based on loan and financing links, while the recycling link is after the use link. In topology, graphics should be centered on use. Loans and financing are parallel, which is used to achieve connectivity, and the finally is recycling link.

Compared with traditional mode, topology rendering in automatic discovery mode is more efficient and intelligent.

\section{Conclusion}

Through the analysis of automatic discovery technology and topology rendering technology of network device, the concept, advantages and implementation methods are discussed. By using these two technologies, we can realize the application value of network equipment, enhance the use performance, and better serve the social life. Under the existing technology, the development trend and objective requirement of the information society are to strive for the realization and popularization of automatic discovery technology and topology rendering technology.

\section{References}

[1]Houben C, Lapkin A A. Automatic discovery and optimization of chemical processes[J]. Current Opinion in Chemical Engineering, 2015, 9:1-7. 
[2]Pignolet Y A, Schmid S, Tredan G. Adversarial topology discovery in network virtualization environments: a threat for ISPs?[J]. Distributed Computing, 2015, 28(2):91-109.

[3]Azodi A, Feng C, Meinel C. Event Driven Network Topology Discovery and Inventory Listing Using REAMS[J]. Wireless Personal Communications, 2015:1-16. 